\title{
The glucocorticoid receptor-FKBP51 complex contributes to fear conditioning and posttraumatic stress disorder
}

\author{
Haiyin Li, ${ }^{1}$ Ping Su, ${ }^{1}$ Terence K.Y. Lai, ${ }^{1}$ Anlong Jiang, ${ }^{1}$ Jing Liu, ${ }^{1}$ Dongxu Zhai, ${ }^{1}$ Charlie T.C. Campbell, ${ }^{1}$ Frankie H.F. Lee, ${ }^{1}$ \\ WeiDong Yong, ${ }^{2}$ Suvercha Pasricha, ${ }^{1,3}$ Shupeng Li, ${ }^{1,3}$ Albert H.C. Wong, ${ }^{1,3,4}$ Kerry J. Ressler, ${ }^{5}$ and Fang Liu ${ }^{1,3,4,6}$ \\ 'Campbell Family Mental Health Research Institute, Centre for Addiction and Mental Health, Toronto, Ontario, Canada. ²omparative Medical Center, Institute of Laboratory Animal Science, Chinese Academy of \\ Medical Sciences \& Peking Union Medical College, Beijing, China. ${ }^{3}$ Department of Psychiatry and ${ }^{4}$ Institute of Medical Science, University of Toronto, Toronto, Ontario, Canada. ${ }^{5}$ McLean Hospital, Harvard Medical \\ School, Belmont, Massachusetts, USA. ${ }^{6}$ Department of Physiology, University of Toronto, Toronto, Ontario, Canada.
}

\begin{abstract}
Posttraumatic stress disorder (PTSD) can develop after exposure to severe psychological trauma, leaving patients with disabling anxiety, nightmares, and flashbacks. Current treatments are only partially effective, and development of better treatments is hampered by limited knowledge of molecular mechanisms underlying PTSD. We have discovered that the glucocorticoid receptor (GR) and FK506 binding protein 51 (FKBP51) form a protein complex that is elevated in PTSD patients compared with unaffected control subjects, subjects exposed to trauma without PTSD, and patients with major depressive disorder (MDD). The GR-FKBP51 complex is also elevated in fear-conditioned mice, an aversive learning paradigm that models some aspects of PTSD. Both PTSD patients and fear-conditioned mice had decreased GR phosphorylation, decreased nuclear GR, and lower expression of 14-3-38, a gene regulated by GR. We created a peptide that disrupts GR-FKBP51 binding and reverses behavioral and molecular changes induced by fear conditioning. This peptide reduces freezing time and increases GR phosphorylation, CR-FKBP52 binding, GR nuclear translocation, and 14-3-3 $\varepsilon$ expression in fear-conditioned mice. These experiments demonstrate a molecular mechanism contributing to PTSD and suggest that the GR-FKBP51 complex may be a diagnostic biomarker and a potential therapeutic target for preventing or treating PTSD.
\end{abstract}

\section{Introduction}

Posttraumatic stress disorder (PTSD) was originally described in soldiers exposed to horrific battlefield events, but the conception of psychological trauma has since been expanded to include any life- or limb-threatening event in either the military or civilian population. PTSD symptoms include flashbacks and nightmares, avoidance of reminders of the trauma, hyperarousal, and insomnia (1). PTSD affects approximately one-fifth of military combat veterans (2) and victims of physical assault (3). Approximately $2 \%$ of US military personnel report having PTSD symptoms (4). In Canada, the lifetime prevalence of PTSD in the general population has been estimated to be $9 \%(5)$.

The current treatment for PTSD is cognitive-behavioral psychotherapy aimed at desensitizing the patient to cues related to the traumatic event through gradual and repeated exposure (6, 7). Medications are generally less effective than therapy, and only 2 medications are currently approved by the US Food and Drug Administration (FDA) for the treatment of PTSD: sertraline and

Authorship note: $\mathrm{HL}$ and PS are co-first authors and they contributed equally to this work.

Conflict of interest: The authors have declared that no conflict of interest exists. Copyright: (5) 2020, American Society for Clinical Investigation.

Submitted: May 17, 2019; Accepted: October 30, 2019; Published: January 13, 2020

Reference information: J Clin Invest. 2020;130(2):877-889.

https://doi.org/10.1172/JCl130363. paroxetine, both of which are selective serotonin reuptake inhibitor antidepressants (8). Unfortunately, overall functioning and outcomes for patients with PTSD remain poor $(9,10)$, and stable remission of chronic PTSD is rare (11). PTSD is unusual among psychiatric disorders in having a clear precipitant, followed by a delay in onset of symptoms, and this interval presents a promising opportunity for preventative interventions in a readily identifiable at-risk population.

The molecular mechanisms underlying PTSD remain largely unknown, but an emerging pathway involves the FK506 binding protein 5 (FKBP5) gene $(12,13)$. FKBP5 is a negative-feedback regulator of the glucocorticoid receptor (GR), so high levels of FKBP5 reduce available GRs and promote glucocorticoid resistance. A specific FKBP5 gene variant (rs1360780) affects susceptibility to PTSD after early-life trauma through modifying GR binding to this gene $(14,15)$. This risk allele likely influences the interaction of the GRE with the promoter, which in turn leads to demethylation of an intron $7 \mathrm{CpG}$ site in FKBP5, resulting in persistent FKBP5 activation (12). The glucocorticoid release triggered by traumatic events in adulthood further activates the demethylated form of FKBP5 and leads to glucocorticoid resistance, which is believed to contribute to the symptoms of PTSD through promoting hyperarousal of the stress-response system (16).

It has been hypothesized that the FKBP51 protein can bind to the GR and sequester it in the cytoplasm $(13,17-19)$, and here we provide direct evidence of such an interaction. We hypothesized 
A

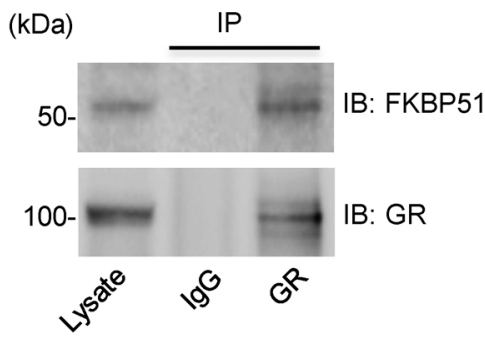

C

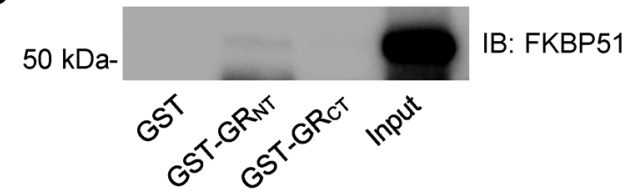

E

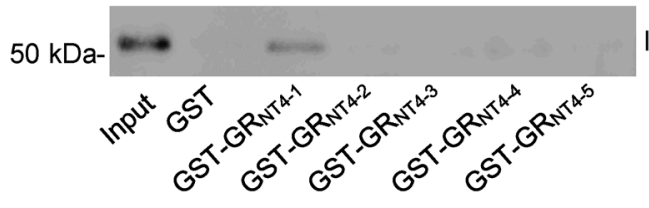

G

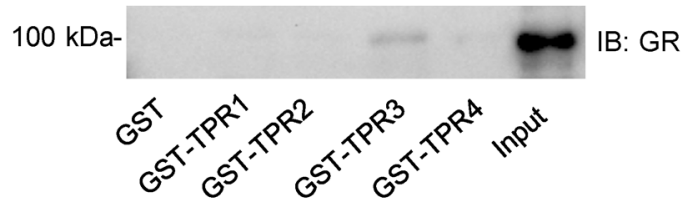

B

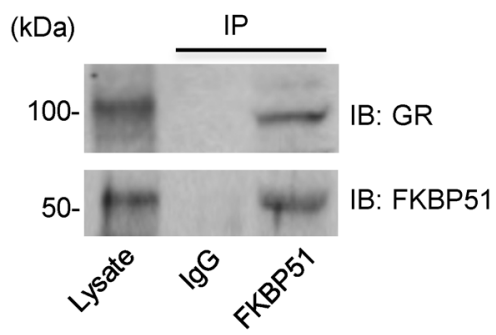

D

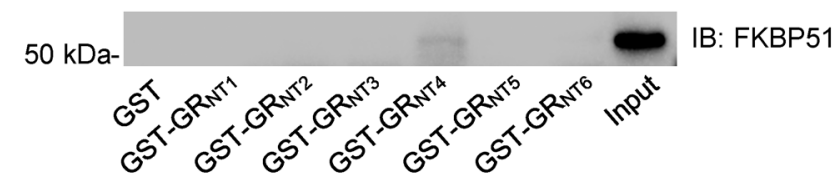

$\mathbf{F}$

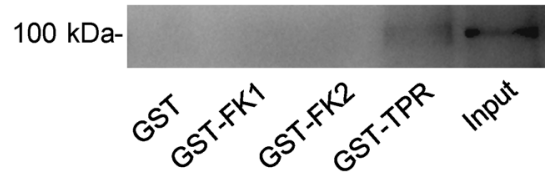

IB: GR

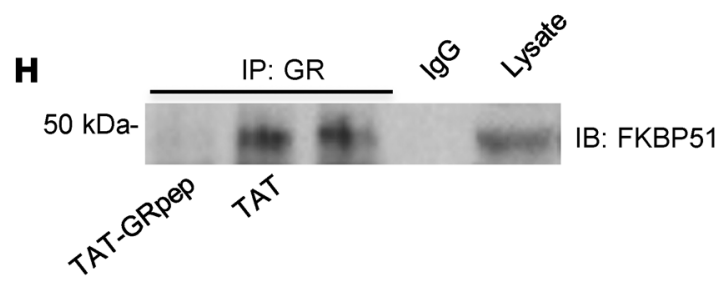

Figure 1. GR forms a complex with FKBP51 via the S211-L225 region of the amino-terminus of CR. (A) In mouse brain lysate, GR antibody, but not IgG (negative control), coimmunoprecipitated with FKBP51. (B) In mouse brain lysate, FKBP51 antibody, but not IgG (negative control), coimmunoprecipitated with GR. (C) Western blot showing that GST-GR ${ }_{\mathrm{NT}}$, but not GST-GR ${ }_{\mathrm{CT}}$, can "pull-down" FKBP51 in mouse brain tissue. (D) Western blot showing that GST-

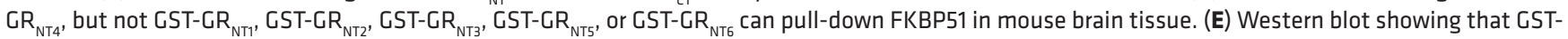

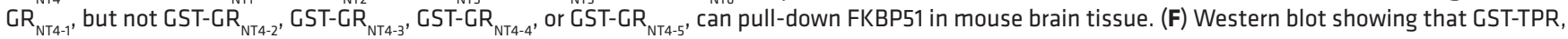
but not GST-FK1 or GST-FK2, can pull-down GR in mouse brain tissue. (G) Western blot showing that GST-TPR3, but not GST-TPR1, GST-TPR2, or GST-TPR4 can pull-down GR in mouse brain tissue. (H) Coimmunoprecipitation shows that TAT-GRpep, but not TAT, is able to disrupt the GR-FKBP51 complex in mouse brain slices. Blots represent 3 independent experiments performed.

that the GR-FKBP51 protein complex should be higher in patients with PTSD and in fear-conditioned mice. If this is correct, a peptide that can disrupt the FKBP51-GR interaction should substantially block all the changes associated with the elevated GR-FKBP51 protein complex and attenuate behavioral responses in mice exposed to strong fear-inducing stimuli. These experiments may demonstrate a mechanism contributing to PTSD, and identify a new treatment target for PTSD.

\section{Results}

GR and FKBP51 form a protein complex in mouse brain. We first demonstrated that FKBP51 forms a protein complex with GR in mouse brain. As shown in Figure 1A, a GR antibody, but not IgG, coimmunoprecipitated with FKBP51, while the FKBP51 antibody coimmunoprecipitates with GR (Figure 1B), suggesting the existence of a GR-FKBP51 complex. The specificity of the FKBP51 antibody was confirmed using proteins extracted from FKBP51 knockout mice (brain tissue provided by WeiDong Yong) $(20,21)$ with FKBP52 as a positive control (Supplemental Figure 1A; supplemental material available online with this article; https://doi.
org/10.1172/JCI130363DS1). As previous studies have indicated that HSP90 (heat shock protein 90) may also form a complex with GR and FKBP51 (22), we confirmed the existence of a GR-HSP90 complex in our experimental conditions. As shown in Supplemental Figure 1B, a GR antibody, but not IgG, coimmunoprecipitated with HSP90, while the HSP90 antibody coimmunoprecipitated with GR in the protein extract from mouse brain.

GR interacts with FKBP51 through the $G R_{N T 4-1}\left(S_{211}-L_{225}\right)$ region. To characterize the physiological and potentially pathological functions of the GR-FKBP51 interaction, we sought to develop an interfering peptide by identifying the regions of the GR that are necessary to bind FKBP51. We initially used GST-fusion proteins encoding the amino-terminus $(\mathrm{NT})$ of $\mathrm{GR}\left(\mathrm{GR}_{\mathrm{NT}}\right)$ and the carboxyl-terminus (CT) of $\mathrm{GR}\left(\mathrm{GR}_{\mathrm{CT}}\right)$ for affinity purification. As shown in Figure 1C, GST-GR $\mathrm{NT}_{\mathrm{NT}}$, but not GST-GR $\mathrm{CT}_{\mathrm{CT}}$, precipitates FKBP51, suggesting that $\mathrm{GR}_{\mathrm{NT}}$ is sufficient for the FKBP51-GR interaction. Extending this strategy, we examined $\mathrm{GR}_{\mathrm{NT}}$ fragments (Supplemental Figure 2) and concluded that $\mathrm{GR}_{\mathrm{NT4} \text {-1 }}$ is the region interacting with FKBP51 (Figure 1, D and E). Similarly, we showed that the TPR (tetratricopeptide repeat) domains of FKBP51 bind 
A

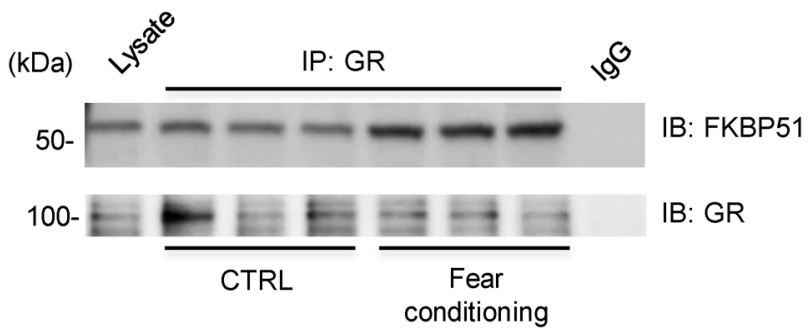

B

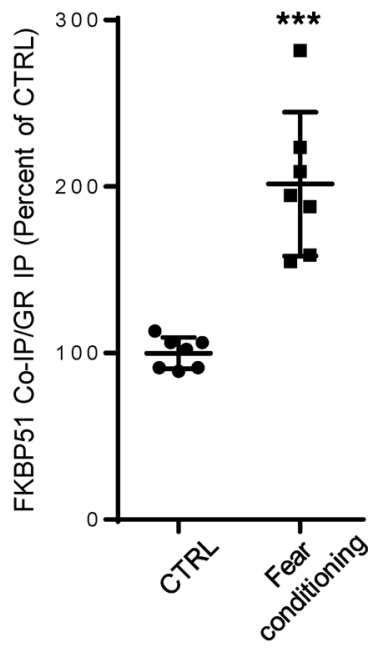

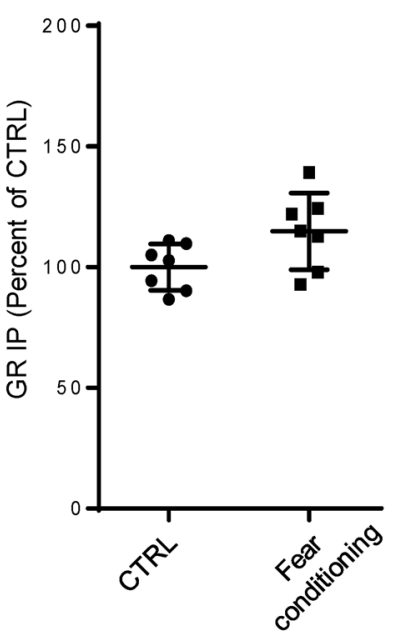
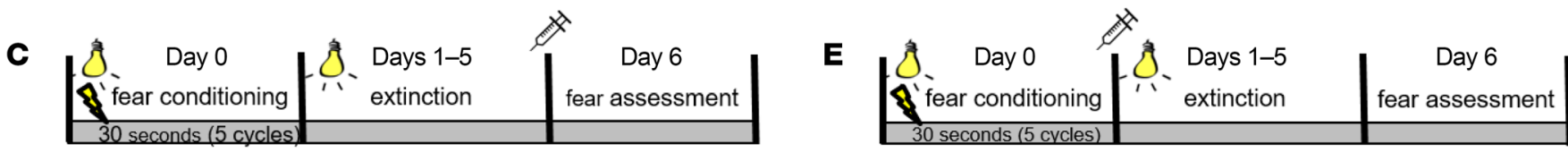

D

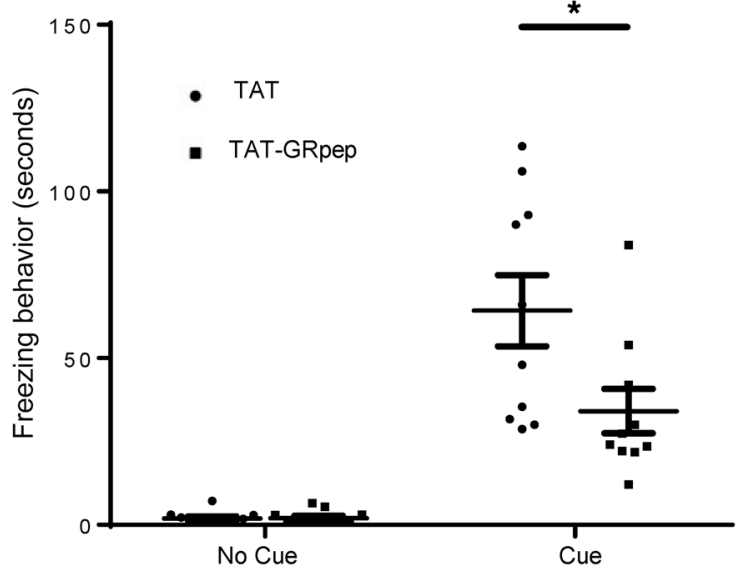

$\mathbf{F}$

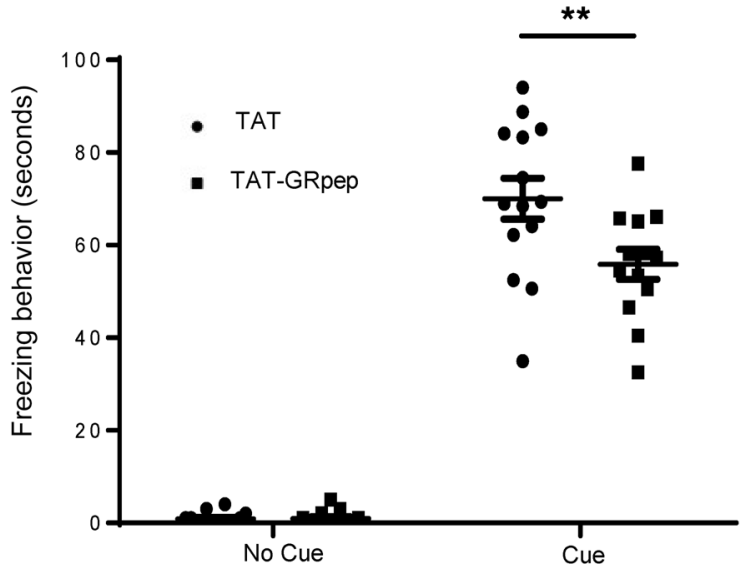

Figure 2. Systemic administration of TAT-GRpep reduces freezing behavior. (A and B) GR-FKBP51 complex levels are significantly higher in brain tissues from fear-conditioned mice. Coimmunoprecipitation shows higher levels of the GR-FKBP51 complex in fear-conditioned mouse brain lysate as compared with control (CTRL) mice. (A) Representative Western blot of FKBP51 and GR precipitated by GR antibody. (B) Densitometric analysis of the levels of FKBP51 coimmunoprecipitated by GR antibody in brain lysate of control (CTRL) or fear-conditioned mice. The level of coimmunoprecipitated FKBP51 (FKBP51 Co-IP) was normalized after being divided by the level of precipitated GR (GR IP). Results for each sample are presented as the percentage of control (CTRL). ${ }^{* *} P<0.001$ as compared with control samples, $n=7, t$ test. Data are shown as mean $\pm \mathrm{SEM}$. (C) A schematic illustration of the experimental schedule regarding data shown in $\mathbf{D}$. (D) During the 3-minute habituation phase (absence of $C S$ ), the animals from both groups displayed a virtual absence of freezing behavior $(P=N S)$. When cues were presented, however, animals in the treatment peptide group froze less than the control peptide (TAT) group $(P<0.05) ; n=10$. (E) A schematic illustration of the experimental schedule regarding data shown in $\mathbf{F}$. (F) TAT-GRpep reduced cued freezing behavior in mice $(P<0.05) ; n=14$ (TAT) or $n=13$ (TAT-GRpep).

GR (Figure 1F). Further dissecting the FKBP51 $1_{\mathrm{TPR}}$ into smaller fragments (Supplemental Figure 3) revealed that FKBP51 ${ }_{\text {TPR3 }}$ is the region interacting with GR (Figure 1G). We synthesized a peptide emulating the amino acid sequence of $\mathrm{GR}_{\mathrm{NT4} 4-1}\left(\mathrm{GRpep}\left[\mathrm{S}_{211}-\mathrm{L}_{225}\right]\right)$ and fused it to the cell-membrane transduction domain of the human immunodeficiency virus-type 1 TAT protein as previously described (23-25). If the $\mathrm{GR}_{\mathrm{NT} 4-1}$ region is essential for GR-FKBP51 binding, GRpep should disrupt the GR-FKBP51 complex by competing with GR for FKBP51. As expected, TAT-GRpep, but not the control TAT peptide, reduced GR-FKBP51 complex levels in mouse brain (Figure 1H). Thus, we conclude that the $\mathrm{GR}_{\text {NT4-1 }}$ region is necessary for GR-FKBP51 complex formation and that GRpep disrupts the GR-FKBP51 complex.

Higher FKBP51-GR complex levels in fear-conditioned mice. We examined whether the GR-FKBP51 complex is affected by fear-conditioning in the mouse, an animal model of aversive learning that simulates some aspects of PTSD (26-28). Cued fear conditioning consisted of 5 pairings of a light and tone (conditioned stimulus [CS]) with a foot-shock (unconditioned stimulus [US]). As shown in Figure 2, A and B, coimmunoprecipitation of FKBP51 
A

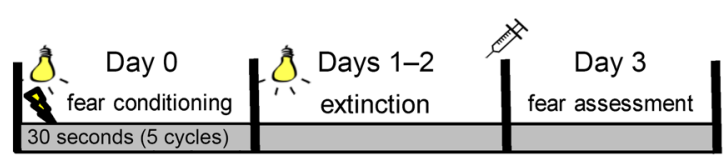

B

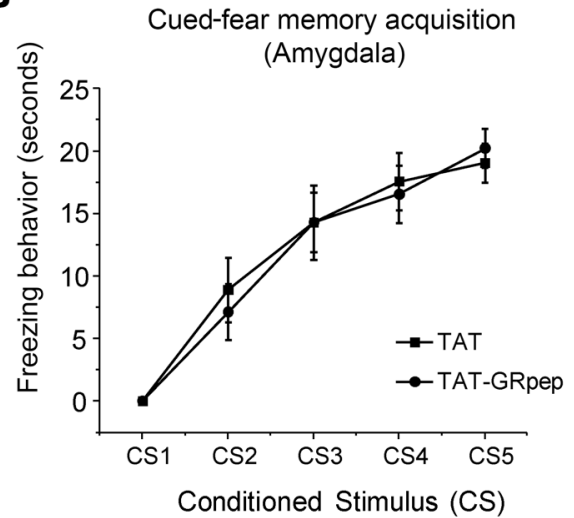

D

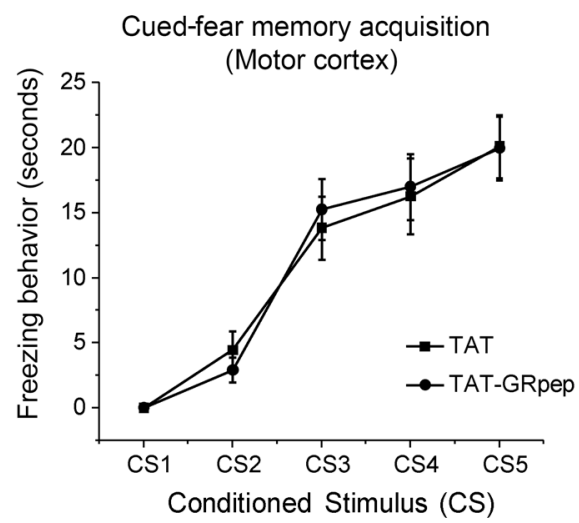

C

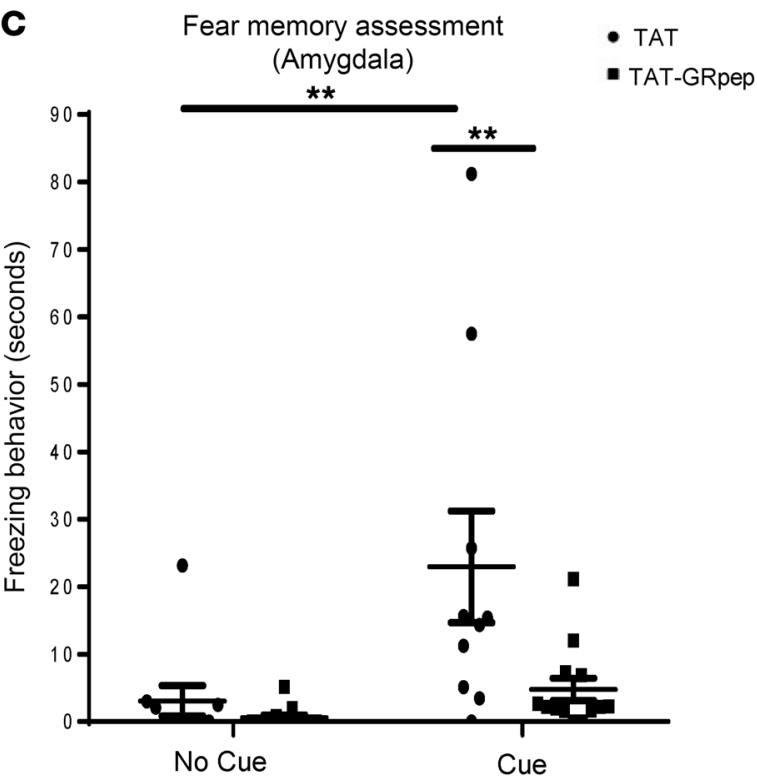

E

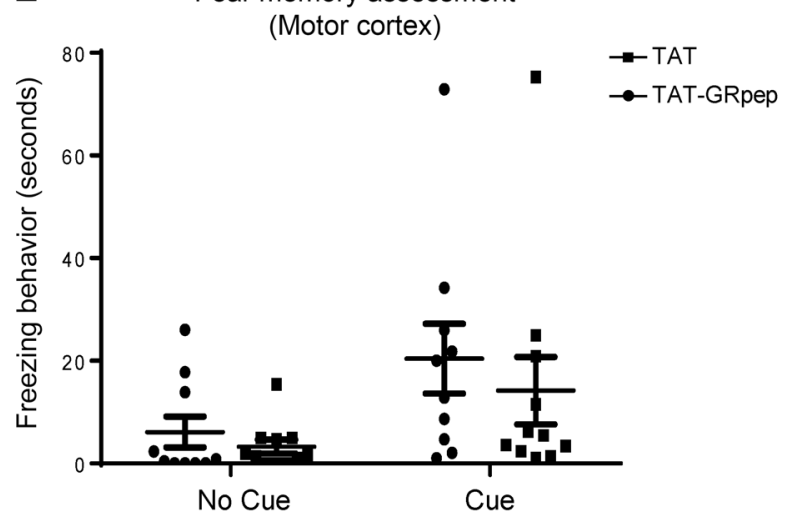

Figure 3. Injection of TAT-GRpep directly into amygdala reduces fear memory behavior. (A) A schematic illustration of the experimental schedule. (B) The 2 groups did not differ in the acquisition of cued-fear memory (2-way ANOVA with repeated measures, $\left.F_{1,21}=0.25, P=N S\right)$. (C) Two-way ANOVA confirmed significant cue effect $\left(F_{1,46}=10.387, P<0.01\right)$, and treatment effect $\left(F_{1,46}=7.708, P<0.01\right)$. The fear memory assessment showed that the treatment peptide (TAT-GRpep) reduced cued-fear memory as the animals injected with TAT-GRpep into the amygdala showed significantly lower level of freezing behavior compared with their control counterparts $(P<0.01) ; n=10$ (TAT) or $n=13$ (TAT-GRpep). (D) For motor cortex injections, the 2 groups did not differ in the acquisition of cued-fear memory (2-way ANOVA with repeated measures $(P=N S)$. (E) The 2-way ANOVA confirmed no significant treatment effect of motor cortex injection $\left(\mathrm{F}_{1,20}=0.353, P=\mathrm{NS}\right)$. The 2 treatment groups did not differ in freezing behavior whether the cue was on or off $(P=\mathrm{NS}$ in both cases); $n=10$ (TAT) or $n=11$ (TAT-GRpep).

by the GR antibody was significantly higher in fear conditioned mice versus control mice $(P<0.001, n=7$, Student's $t$ test, power $=0.993)$. There is no significant difference in direct immunoprecipitation of GR between the 2 groups (Figure 2B), suggesting that the GR antibody precipitates equal amounts of GR in both groups.

Systemic TAT-GRpep delivery reduces freezing. If the GR-FKBP51 complex is part of the mechanism by which fear memories are stored or expressed, disrupting the complex should interfere with these memories. Thus, we tested the hypothesis that our GR-FKBP51-interfering peptide should reduce freezing behaviors in fear-conditioned mice. Two randomized groups of animals underwent 5 rounds of CS-US pairings (CS = light, US $=0.5 \mathrm{~mA}$ foot shock for 1 second). The animals were then subjected to 5 days of extinction, in which the same cues were presented without foot shock, before cued fear memory was assessed (Figure 2C). Approximately 2 hours before assessing the fear memory, animals were injected intraperitoneally (i.p.) with either control (TAT) or treatment peptide (TAT-GRpep) (3 nmol/g, i.p.). As shown in Figure 2D, there was no difference in freezing behavior between the TAT-GRpep and TAT alone groups during the habituation period without CS ( $P=\mathrm{NS})$. However, with the CS, TAT-GRpep significantly reduced freezing compared with the TAT peptide (34.1 \pm 6.7 seconds vs. $64.2 \pm 10.7$ seconds, 2 -way ANOVA with repeated measures, $\left.\mathrm{F}_{1,18}=5.988, P<0.05\right)$, suggesting that TAT-GRpep can block the emergence of PTSD-like fear-related behaviors.

Furthermore, we tested whether disrupting the GR-FKBP51 complex reduces consolidation of cued fear memory. Thus, TAT or TAT-GRpep was administered 2 hours after the end of 
A

$(\mathrm{kDa})$

100-

$100-$

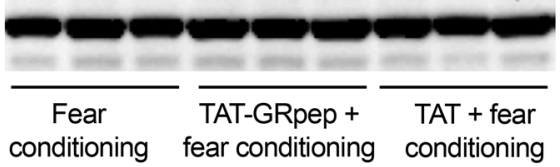

B: GR

C

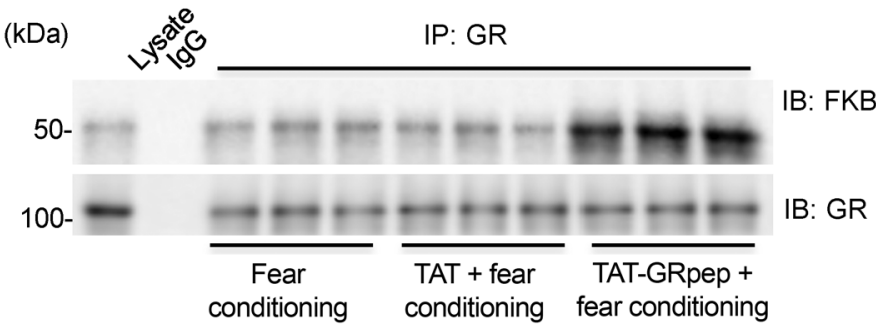

E

$(\mathrm{kDa})$

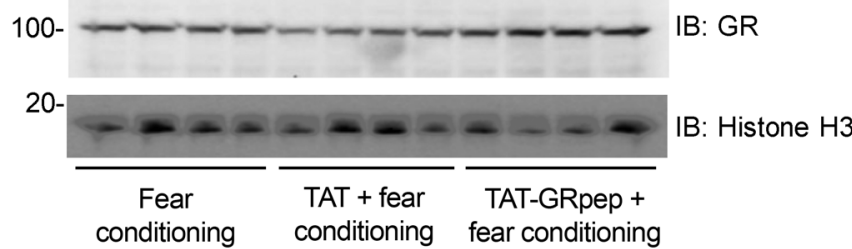

G

(kDa)

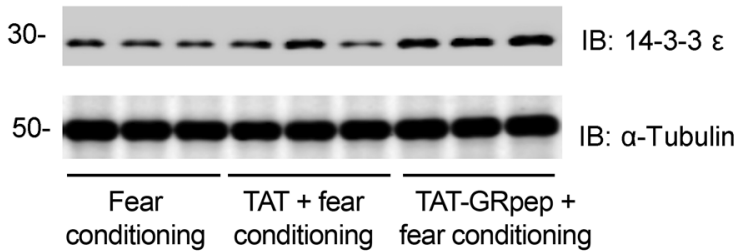

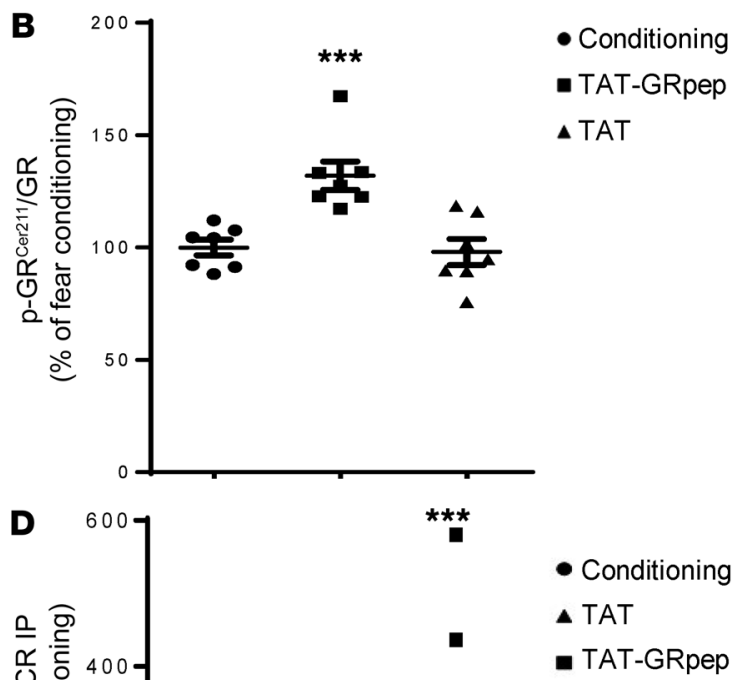

- TAT-GRpep
$\boldsymbol{\Delta}$

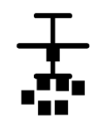

犬̊

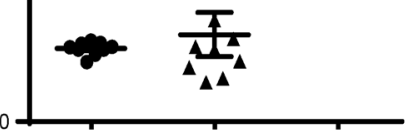

\section{F}

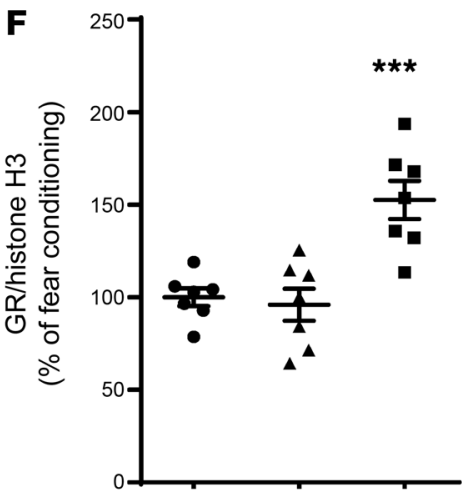

- Conditioning

$\triangle$ TAT

-TAT-GRpep

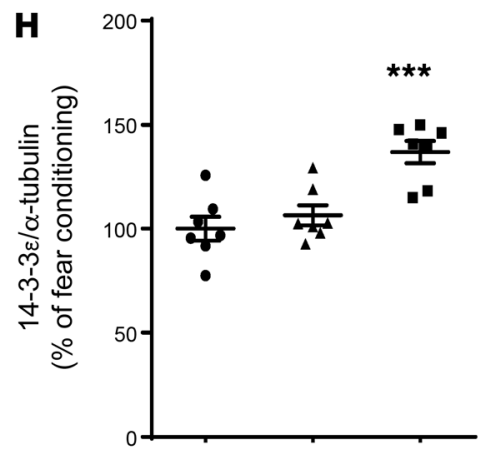

- Conditioning

\ TAT

- TAT-GRpep

Figure 4. Disruption of GR-FKBP51 complex affects GR signaling in fear-conditioned mice. Representative Western blots (A) and densitometric analysis (B) of the levels of phosphorylated GR $\left(\mathrm{S}_{211}\right)$ in brain lysate from fear-conditioned mice injected with saline, TAT, or TAT-CRpep. The level of phosphorylated GR $\left(\mathrm{S}_{211}\right)$ was normalized after being divided by the level of GR. ${ }^{* *} P<0.001, n=7, F_{2.18}=12.81$, 1-way ANOVA test followed by Dunnett's post hoc test. (C-D) Representative Western blots (C) and densitometric analysis (D) of the levels of FKBP52 coimmunoprecipitated by GR antibody in fear-conditioned mice injected with saline, TAT-GRpep, or TAT peptide (3 nmol/g i.p.). The level of coimmunoprecipitated FKBP52 (FKBP52 Co-IP) was normalized after being divided by the level of precipitated GR (GR IP). ${ }^{* *} P<0.001$ as compared with the fear-conditioned sample, $n=9, F_{2,24}=14.15$, 1-way ANOVA test followed by Dunnett's post hoc test. (E-F) Representative Western blots $(\mathbf{E})$ and densitometric analysis $(\mathbf{F})$ of levels of nuclear GR from fear-conditioned mice injected with saline, TAT, or TAT-CRpep ( 3 nmol/g i.p.). The level of nuclear GR was normalized after being divided by the level of Histone $H 3$. ${ }^{* * *} P<0.001$ as compared with the fear-conditioned group, $n=$ $7, F_{2,18}=14.50$, 1-way ANOVA test followed by Dunnett's post hoc test. (G-H) Representative Western blots $(\mathbf{G})$ and densitometric analysis (H) of expression levels

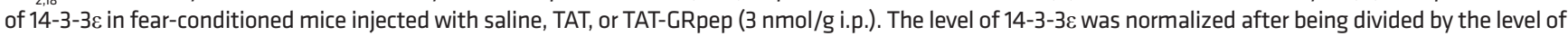
$\alpha$-Tubulin. ${ }^{* *} P<0.001$ as compared with the fear-conditioned group, $n=7, F_{2,18}=13.57,1$-way ANOVA test followed by Dunnett's post hoc test. For densitometric analysis, results are presented as the percentage of the fear-conditioned samples. Data are shown as mean \pm SEM. 
fear conditioning (Figure 2E), before 5 consecutive daily extinction sessions of cue exposure without shock. Twenty-four hours after the last extinction session, neither treatment group froze without the CS. With the CS, the TAT-GRpep treatment group had significantly less freezing compared with the control TAT group $(55.9 \pm 3.3$ seconds vs. $71.0 \pm 3.6$ seconds, 2-way ANOVA with repeated measures, $F_{1,25}=6.007, P<0.05$, Figure $2 \mathrm{~F}$ ). The ability of TAT-GRpep to disrupt the GR-FKBP51 complex in fear-conditioned mice was confirmed with coimmunoprecipitation in both brain tissues (Supplemental Figure 4, A and B, 1-way ANOVA, $\mathrm{F}_{2,18}=23.61, P<0.001, n=7$, power $\left.=0.984\right)$ and peripheral blood samples (Supplemental Figure 4, C and D, $P<$ 0.01, $n=7$, Student's $t$ test, power $=0.992)$. We also confirmed the presence of TAT-GRpep in brain using confocal fluorescent microscopy (Supplemental Figure 4E) and in blood using flow cytometry (Supplemental Figure 4F). These results suggest that disruption of the GR-FKBP51 complex blocks the expression of PTSD-like fear-related behaviors, and inhibits the consolidation of cued fear memory.

Direct injection of TAT-GRpep into amygdala reduces freezing. To better understand where the GR-FKBP51 complex is acting to modulate fear memory, we tested whether direct injection of TAT-GRpep into the right amygdala would have effects similar to systemic injection (Figure 3A). The amygdala was targeted because it is critical for fear and emotional learning (29). As shown in Figure 3B, injection with either TAT-GRpep or the TAT control peptide produced no differences in the acquisition of cued-fear memory (2-way ANOVA with repeated measures, $\left.\mathrm{F}_{1,21}=0.25, P=\mathrm{NS}\right)$. However, 2-way ANOVA confirmed a significant effect of the cue $\left(F_{1,46}=10.387, P<0.01\right)$ and peptide $\left(\mathrm{F}_{1,46}=7.708, P<0.01\right)$ during the recall phase of fear memory testing. These results show that TAT-GRpep attenuated cued-fear memory expression since these animals had significantly less freezing than controls ( $P<0.01$, Figure $3 C)$. To demonstrate the anatomical specificity of TAT-GRpep effects, we repeated the same experiments with peptide infusions into the motor cortex instead of the amygdala. As shown in Figure 3D, both groups acquired the memory of cued fear (2-way ANOVA with repeated measures, $F_{1,20}$ $=0.03, P=\mathrm{NS})$. Injecting TAT-GRpep into the motor cortex did not affect freezing $\left(F_{1,20}=0.353, P=N S\right)$, nor was there an interaction effect $\left(\mathrm{F}_{1,20}=0.293, P=\mathrm{NS}\right)$. Both treatment groups reacted similarly to the cue ( $P=$ NS, Figure $3 \mathrm{E}$ ). These data show that injection of TAT-GRpep specifically into the amygdala is sufficient to block the emergence of PTSD-like fear behaviors in the mouse.

TAT-GRpep peptide affects GR signaling in fear-conditioned mice. The next objective was to study the effects of the TATGRpep peptide on GR signaling by investigating whether disruption of the GR-FKBP51 complex affects GR phosphorylation, the GR-FKBP52 (aka FKBP4) interaction, and GR nuclear translocation $(30,31)$. In general, phosphorylation of GR protects it from degradation $(32,33) . S_{211}$ is a known GR phosphorylation site located within the GR-FKBP51 interacting region, and $S_{211}$ phosphorylation increases recruitment of GR to target genes (34). Thus, we investigated whether the TAT-GRpep alters GR $\mathrm{S}_{211}$ phosphorylation in fear-conditioned mice. As shown in Figure $4, \mathrm{~A}$ and $\mathrm{B}, \mathrm{GR} \mathrm{S}_{211}$ phosphorylation is significantly higher in fear-conditioned mice treated with TAT-GRpep compared with those treated with TAT control or no treatment ( $n=7,1$-way
ANOVA, $F_{2,18}=12.81, P<0.001$, power $\left.=0.950\right)$. Decreased GR $\mathrm{S}_{211}$ phosphorylation is observed in fear-conditioned mice compared with control mice (Supplemental Figure 5, A and B, $n=7, P$ $<0.01$, Student's $t$ test, power $=0.962$ ).

Previous studies have suggested that impaired GR $\mathrm{S}_{211}$ phosphorylation decreases GR nuclear translocation (35). Others have suggested that dissociation of the GR-FKBP51 complex would promote GR-FKBP52 complex formation, leading to GR nuclear translocation, where GR can activate or repress transcription of target genes (36). We saw that TAT-GRpep increased GR $S_{211}$ phosphorylation in fear-conditioned mice. Thus, we predict that TAT-GRpep should also increase GR-FKBP52 complex formation and GR nuclear expression when administered to fear-conditioned mice. Consistent with our hypothesis, we found significant increases in GR-FKBP52 complex levels in fear-conditioned mice after TAT-GRpep treatment (Figure 4, $\mathrm{C}$ and $\mathrm{D}, 1$-way ANOVA $\mathrm{F}_{2,24}=14.15, P<0.001, n=9$, power $=$ 0.930). GR nuclear localization in fear-conditioned mice was also increased by TAT-GRpep (Figure 4, E and F, 1-way ANOVA, $\mathrm{F}_{2,18}=14.50, P<0.001, n=7$, power $\left.=0.951\right)$.

Our observation that TAT-GRpep peptide treatment increases GR nuclear translocation implies that both GR nuclear translocation and GR-mediated gene transcription should be lower in fear-conditioned mice. Indeed, we found a decreased expression of 14-3-3e, a target protein of GR, in fearconditioned mice $(37,38$ ) (Supplemental Figure 5, C and D, $P<$ $0.01, n=7$, Student's $t$ test, power $=0.967)$. This fear-mediated suppression of $14-3-3 \varepsilon$ is rescued by TAT-GRpep treatment (Figure 4, G and H, 1-way ANOVA, $\mathrm{F}_{2,18}=13.57, P<0.001, n=7$, power $=0.970$ ). Together, these data suggest that disruption of GR-FKBP51 complex with TAT-GRpep increases GR S ${ }_{211}$ phosphorylation, GR-FKBP52 complex levels, and GR nuclear translocation. All of these may contribute to increased expression of the GR-regulated protein 14-3-3 $\varepsilon$ in fear-conditioned mice.

Higher GR-FKBP51 complex levels, decreased GR $S_{211}$ phosphorylation, and reduced nuclear GR in peripheral blood from PTSD patients. The next set of experiments was aimed at translating the fearconditioned mouse results to humans. Of course it is not possible to obtain brain tissue from our patients, and instead we obtained peripheral blood samples. We observed similar results when analyzing GR-FKBP51 levels in either blood or brain tissue from mice (Supplemental Figure 4, A and B vs. C and D). Thus, we argue that examining the GR-FKBP51 interaction and associated changes in peripheral blood of PTSD patients provides useful insights despite the lack of brain tissue for this part of our study. Descriptive statistics and demographic features of our clinical samples are shown in Supplemental Table 1 and Supplemental Table 2.

We measured GR-FKBP51 complex levels in the peripheral blood of PTSD patients recruited from the Centre for Addiction and Mental Health (CAMH), University of Toronto (Supplemental Table 1). Equal amounts of protein from each sample were incubated with anti-FKBP51 antibody and the precipitated proteins were immunoblotted with either FKBP51 or GR antibody. Each Western blot included 5 samples per group and results were normalized against the mean of 5 control samples on the same blot. We found that the GR-FKBP51 complex is significantly higher in peripheral blood from PTSD patients compared with 
A

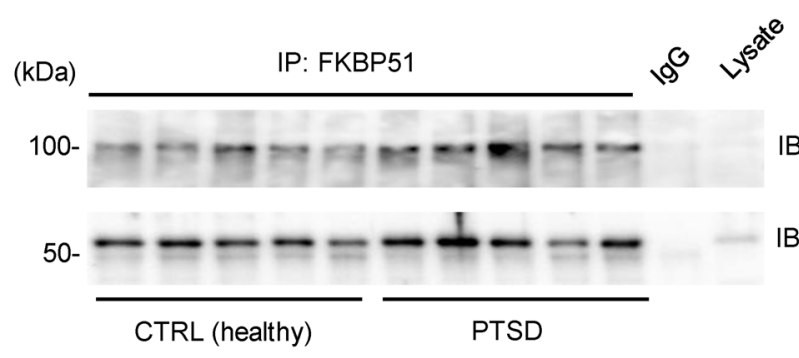

C
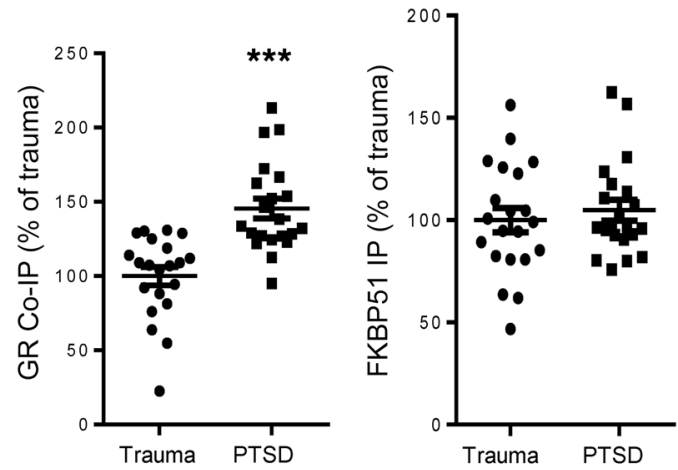

E
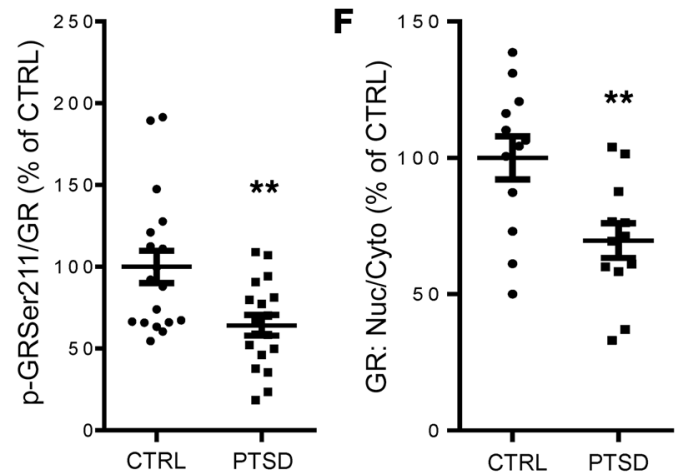

IB: GR

IB: FKBP51
B

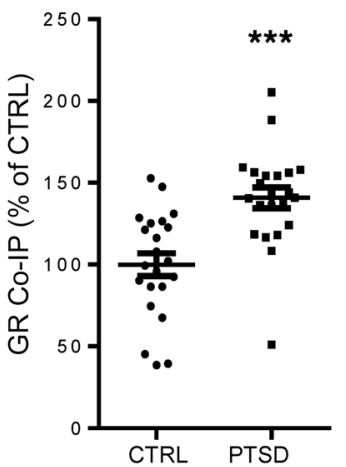

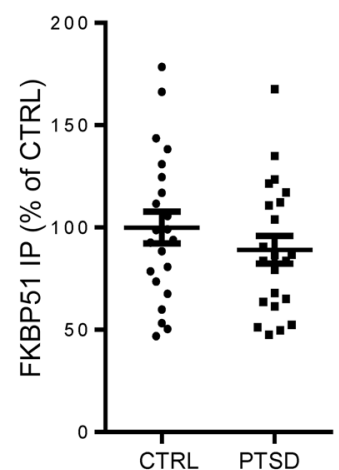

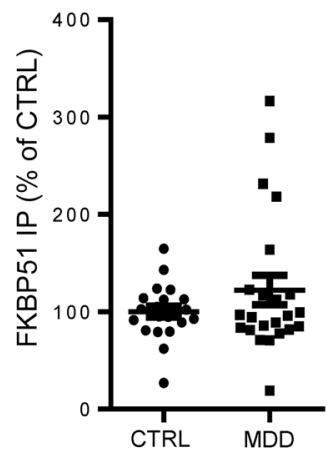

D

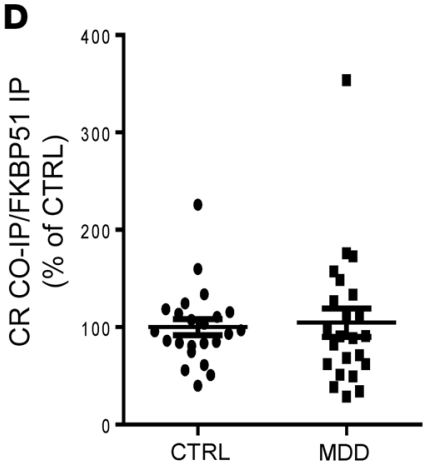

G

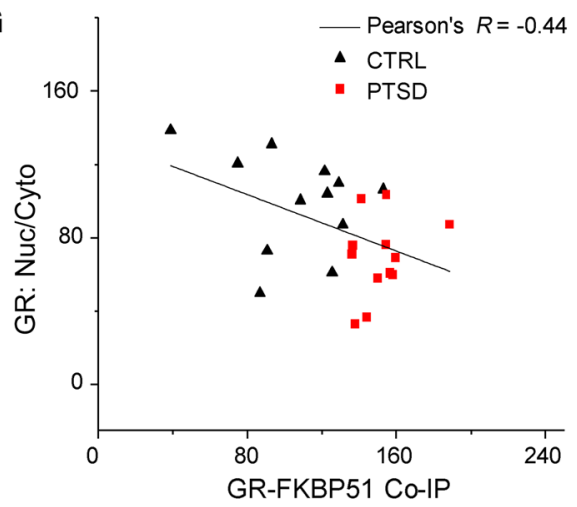

H $(\mathrm{kDa})$

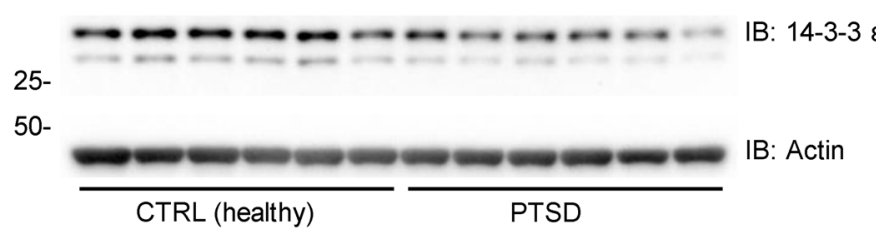

I

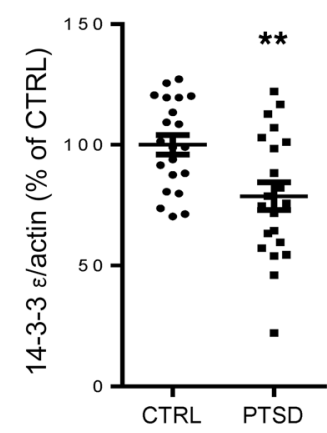

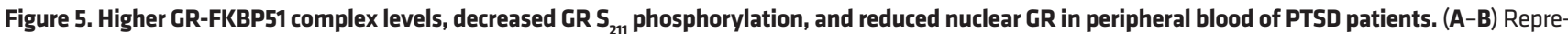
sentative Western blots (A) and densitometric analysis (B) of the levels of CR (left), FKBP51 (right) coimmunoprecipitated by FKBP51 antibody in peripheral blood samples from PTSD patients and healthy controls. Results for each sample are presented as the percentage of the mean of the control samples on the same blot. CTRL: $1 \pm 0.069$, PTSD: $1.408 \pm 0.641,{ }^{* * *} P<0.001, n=22$, $t$ test. (C) Densitometric analysis of the levels of GR (left), FKBP51 (right) coimmunoprecipitated by FKBP51 antibody in peripheral blood samples from PTSD patients and trauma controls. Results are presented as the percentage of trauma controls on the same blot. Trauma: $1 \pm 0.061$, PTSD: $1.455 \pm 0.661,{ }^{* *} P<0.001, n=21, t$ test. (D) Densitometric analysis of the levels of GR (left), FKBP51 (right) coimmunoprecipitated by FKBP51 antibody in peripheral blood samples from MDD patients and healthy controls. Results are presented as the percentage of healthy controls on the same blot; $P=0.7768, n=23, t$ test. (E) Densitometric analysis shows decreased GR phosphorylation at $\mathrm{S}_{211}$ in lymphocytes from PTSD patients compared with healthy controls (CTRL); ${ }^{* *} P<0.01, n=18, t$ test. (F) Densitometric analysis of levels of nuclear expression of GR in lymphocytes from PTSD patients and healthy controls (CTRL); ${ }^{*} P<0.01, n=12, t$ test. (C) FKBP51 binding inhibits nuclear translocation of GR. Reduced nuclear translocation of GR is negatively correlated with higher GR-FKBP51 complex levels in lymphocytes from PTSD patients compared with CTRL; $n=24$ (12 PTSD and 12 CTRL), Pearson correlation coefficient $r=-0.44392, P=$ 0.03. (H-I) Representative Western blots $(\mathbf{H})$ and densitometric analysis (I) of levels of 14-3-3E expression in lymphocytes from PTSD patients and healthy controls (CTRL). Results are presented as the percentage of the mean of the control samples on the same plot. ${ }^{* *} P<0.01, n=21, t$ test. Data are shown as mean \pm SEM. 


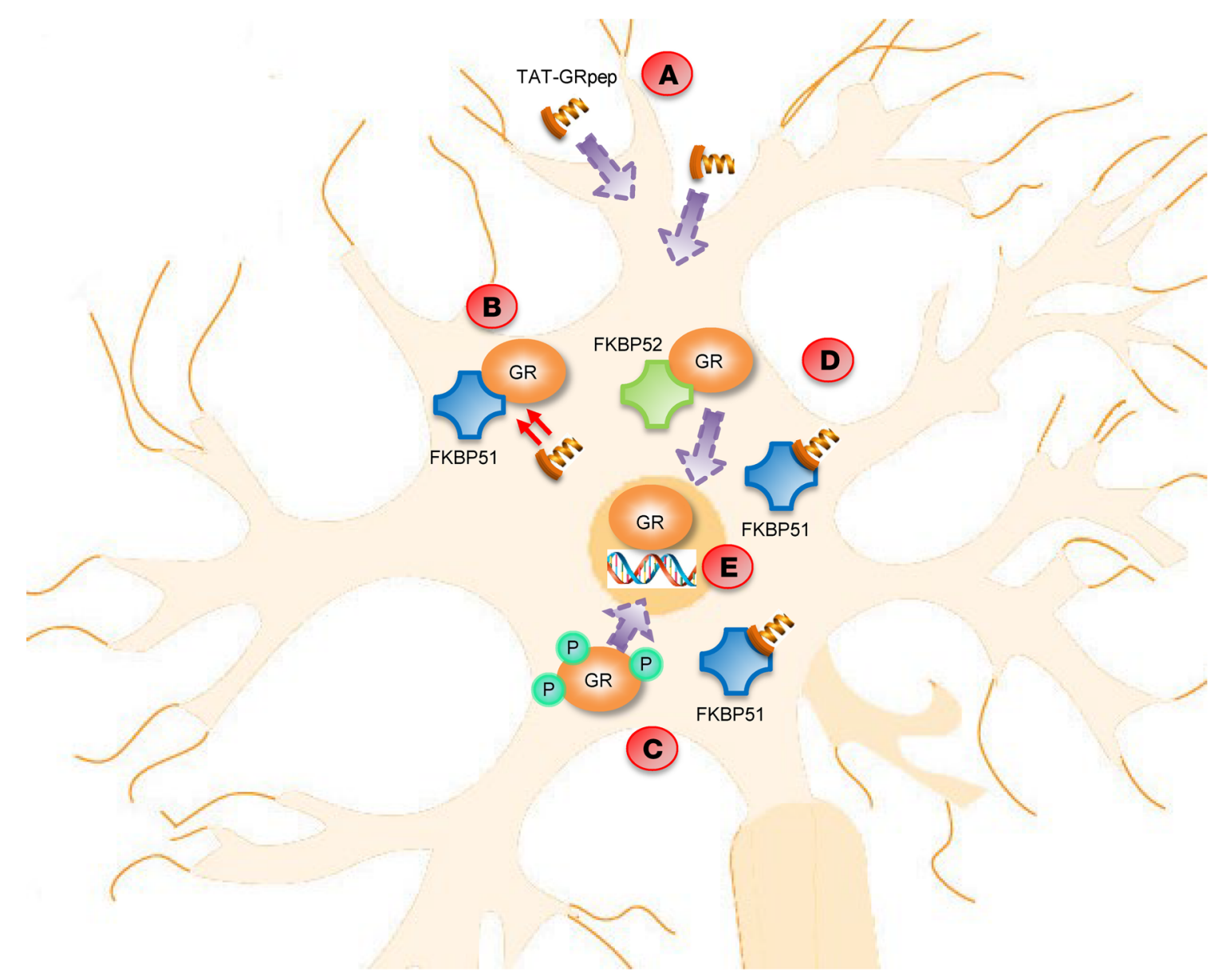

Figure 6. Summary of the molecular pathways within the neuron in the presence of TAT-CRpep. (A) TAT-CRpep is able to pass through the blood brain barrier and enter into the neuron due to the TAT sequence. (B) Once TAT-GRpep enters the cell, it competes with GR in binding to FKBP51. (C) More GR is phosphorylated and (D) consequently, more GR can bind to FKBP52. (E) Both events are responsible for translocating GR into the nucleus, where it binds to specific DNA sequences and promotes transcription.

healthy control subjects $(n=22$ each group; $P<0.001$, Student's $t$ test, power $=0.988$; Figure 5 , A and B). There was no significant difference either in the direct immunoprecipitation of FKBP51 (Figure 5B) or the total expression of FKBP51 (Supplemental Figure $6 \mathrm{~A}$ ) between the 2 groups, suggesting that the observed higher level of GR-FKBP51 complex was not due to the differences in the amount of FKBP51 precipitated by FKBP51 antibody or the total expression of FKBP51 between the 2 groups.

We also obtained evidence that the elevated GR-FKBP51 interaction is specific to PTSD and not just a biomarker of trauma exposure per se, by performing a replication and extension analysis in 42 subjects from the Grady Trauma Project in Atlanta, GA (14, 39, 40). All subjects had significant trauma exposure, but while 21 had severe PTSD, the age-, sex-, and race-matched trauma controls had few symptoms (Supplemental Table 2). We found significantly elevated GR-FKBP51 in peripheral blood from PTSD subjects versus trauma-exposed controls ( $P<0.001$, Student's $t$ test, $n=21$, power $=0.99853$, Figure $5 \mathrm{C}$ ), with no significant difference in direct immunoprecipitation of FKBP51 between the 2 groups (Figure 5C). These combined data suggested that the FKBP51-GR complex might be a biomarker for PTSD. In addition, major depressive disorder (MDD) has some similarities with PTSD in the context of GR signalling that involves FKBP5 $(41,42)$. We also measured FKBP51-GR complex levels in a separate group of samples from patients with MDD from Atlanta, GA. As shown in Figure 5D, there was no difference in FKBP51-GR complex levels between MDD and control subjects, and there was also no difference in FKBP51 expression alone (Supplemental Figure 6B). Descriptive statistics and demographic features of the MDD samples are shown in Supplemental Table 3. These results suggest that MDD does not significantly alter FKBP51-GR complex levels.

Consistent with our observation in fear-conditioned mice, we found that $S_{211}$ phosphorylation is significantly decreased in peripheral blood samples from the Toronto PTSD patients (Figure $5 \mathrm{E}, n=18, P<0.01$, Student's $t$ test, power $=0.835$ ). These patients also had a lower proportion of GR in the nucleus compared with healthy control subjects (Figure 5F, $P<0.01$, $n=12$, power $=0.817$ ). Further analysis suggests that higher GR-FKBP51 complex levels are correlated with a lower proportion of the GR in the nucleus (Pearson's $r=-0.44, P=0.030$, Figure 5G). More importantly, the decreased expression of GR in the nucleus has a functional impact as shown by reduced transcription of $14-3-3 \varepsilon$, a gene regulated by GR $(37,38)$ (Figure 5, $\mathrm{H}$ and $\mathrm{I}, P<0.01, n=21$, power $=0.841)$. These data suggest 
that PTSD patients have elevated GR-FKBP51 complex levels, which may lead to less GR nuclear translocation and decreased GR-mediated gene transcription in PTSD.

\section{Discussion}

In summary, we have direct evidence for a GR-FKBP51 protein complex that is elevated in fear-conditioned mice and PTSD patients. We synthesized a blocking peptide TAT-GRpep, which contains the same amino acid sequence as the region of GR that binds FKBP51. This peptide blocks both the consolidation and recall of fear memories in mice. We propose that the reduction of PTSD-related fear behaviors by TAT-GRpep begins with the dissociation of GR-FKBP51, which leads to greater GR S ${ }_{211}$ phosphorylation and more GR-FKBP52 complex, both of which facilitate GR nuclear translocation (working model shown in Figure 6). Restoring GR to the nucleus in turn rescues the deficient transcription of GR-regulated target genes such as 14-3-3e.

Although the GR-FKBP51 complex is elevated in our subset of patients with PTSD, we are not suggesting that the GR-FKBP51 complex is pathological per se. PTSD can be conceptualized as a failure to recover from the normal acute reaction to trauma, in which patients cannot return to a physiological homeostasis (43). In this model, there should be a spike in GR-FKBP51 levels immediately after trauma, but PTSD arises in conjunction with a failure to return GR-FKBP51 to normal levels over time. The fact that the GR-FKBP51 complex is present in control subjects suggests that this complex has a physiological role in healthy individuals. Indeed, many studies support the view that the GR-FKBP51 interaction has protective effects via FKBP51 suppression of GR signaling $(43,44)$. Thus, the GR-FKBP51 complex has an important normal physiological function but in excess it could be pathological. This seems to be a more general phenomenon that we have observed in our previous studies with other protein complexes in brain disorders $(23,45,46)$.

One of the main claims of this paper is that the elevated GR-FKBP51 complex is specific to PTSD. We base this conclusion on our data showing that the GR-FKBP51 complex is not increased in subjects exposed to trauma who did not develop PTSD (Figure 5C), nor in a separate cohort of patients with MDD alone (Figure 5D). Future research could examine GR-FKBP51 levels in other neuropsychiatric disorders, especially those with an anxiety and fear-related component, to further establish the specificity of this protein complex as a biomarker for PTSD. Cortisol is a critical component of the stress response, and any discussion of molecular mechanisms in PTSD must include this hormone. Currently, there is no consensus in the literature about cortisol levels in PTSD, in part because patients with PTSD are very heterogeneous and because symptoms fluctuate in a given patient. There is a substantial body of research reporting lower circulating levels of cortisol in PTSD, but a similar amount of evidence supports the hypothesis of enhanced negative feedback or enhanced GR sensitivity in PTSD (for review, see ref. 43). Subgroups of patients may have increased, decreased, or apparently normal levels of cortisol. Patient heterogeneity could be due to preexisting genetic or epigenetic factors affecting FKBP51 transcription in response to a traumatic event (12). Patients also vary as to the type and pattern of trauma (chronic vs. single acute event), premorbid and ongoing psychiatric comorbidity (e.g. other anxiety or mood disorders, substance abuse), the timing of sample collection relative to the trauma, and symptoms present at the time of sample collection. Thus, the issue of HPA axis activation in relation to PTSD and conditioned fear behaviors is complex.

In addition, we are aware that previous studies of PTSD found increased suppression of endogenous cortisol release by dexamethasone $(47,48)$, a standard indicator of reduced response to glucocorticoids, suggesting that PTSD is associated with enhanced GR signaling. There is also data supporting the notion that insufficient glucocorticoid signaling is present in stress-related psychiatric disorders, including PTSD $(49,50)$. An important issue here is the utility of the dexamethasone suppression test (DST), which was classically used as a test for depression but has since been abandoned (51). There are several reasons why the DST is no longer used as a clinical assay in evaluating depression, including the variable bioavailability of dexamethasone and the unreliability of the cortisol assay procedure. As well, the DST does not reliably distinguish depression from other diagnoses. It is also important to note that the DST is most consistently abnormal in Cushing's syndrome, which is certainly not the clinical picture in PTSD, nor in depression.

We did not observe significant changes in cortisol levels in our group of PTSD patients, who are mostly females with chronic or repeated trauma. In our mouse model, we measured the change in glucocorticoid levels at 0.5, 2, and 24 hours after fear conditioning in mice. There is a significant increase in corticosterone 0.5 hour after fear conditioning compared with control animals, but not at 2 and 24 hours after fear conditioning (Fang Liu, unpublished observations). Thus, it seems that glucocorticoid levels increase immediately after exposure to a fearful stimulus, but subside very quickly. While fear conditioning in the mouse is not a perfect model for PTSD in humans, the normal cortisol level in our PTSD patients could still be consistent with our claim that the GR-FKBP51 complex is involved in PTSD in humans and fear-conditioned learning in the mouse.

There are some apparent inconsistencies between our data and the literature with regard to the levels of FKBP51, independent of the GR-FPBP51 complex. Increased FKBP5 mRNA and FKBP5 methylation have been reported to be elevated in MDD, PTSD, and with childhood trauma (12, 41, 52-54). However, in our study, we found no difference in total FKBP51 protein expression between PTSD and control subjects (Supplemental Figure 6A). This is not a direct contradiction, since mRNA levels, gene methylation, and protein expression have a complex relationship and can often be divergent. We did observe a nonsignificant trend toward higher FKBP51 protein levels in patients with MDD alone compared with control subjects (Supplemental Figure 6B), which was consistent with earlier mRNA data. However, the amount of GR-FKBP51 complex was not different between MDD and unaffected control subjects (Figure 5D).

Although previous studies have reported that GR, HSP90, and FKBP51 form a protein complex (55), they suggest that GR and FKBP51 interact indirectly via HSP90. Those other studies also suggest that the GR interacts with HSP9O via its hormone binding domain (HBD), located in the carboxyl terminal region of GR $(56,57)$. In contrast, our data show that the NT end of GR interacts 
with FKBP51, and so it appears that different domains of GR bind to FKBP51 and HSP90. It is possible for there to be multiple interacting sites between 2 proteins, as we showed previously with the dopamine D1 and NMDA receptors (58).

We relied on a classic technique for measuring protein interactions: coimmunoprecipitation. With this method, it is critical to ensure that equal amounts of protein are precipitated by the primary antibody. For samples from PTSD patients, we deliberately used excess protein to saturate the limited amount of anti-FKBP51 antibody, resulting in approximately equal amounts of immunoprecipitated FKBP51 protein (Figure 5, A-D). We were also careful to add equal amounts of protein from each patient sample into the primary antibody mixture and to equally divide the precipitated proteins for immunoblots with either GR or FKBP51 antibody. Thus, our results suggest increased GR-FKBP51 levels in PTSD when equal amounts of FKBP51 are present.

One of the key issues in our project is whether the effect of the TAT-GRpep is specific to the GR-FKBP51 interaction. The TAT-GRpep sequence was derived from the region within GR that is responsible for binding to FKBP51. We entered the GRpep $\left[\mathrm{S}_{211}-\mathrm{L}_{225}\right]$ sequence into protein BLAST and did not find any proteins with high homology, including the related mineralocorticoid receptor (MR) $(59,60)$. Coimmunoprecipitation of both GR-FKBP51 and GR-FKBP52 in mouse brain lysates shows that TAT-GRpep reduces GR-FKBP51 binding (Figure 1H, Supplemental Figure 4, A-D), but not GR-FKBP52 binding (Figure 4, C and D), demonstrating good selectivity. Homology analysis of the FKBP51 region (TPR3) that interacts with GR (Figure $1 \mathrm{G}$ ) shows only $44 \%$ amino acid homology with the corresponding region of FKBP52. Thus, TAT-GRpep is likely specific to the GR-FKBP51 interaction.

Another important consideration is whether the interfering peptide TAT-GRpep actually was present in the tissues being analyzed and whether the peptide was successful in disrupting the GR-FKBP51 complex. We used fluorescence microscopy to visualize TAT-GRpep labelled with anti-TAT antibodies, and showed that TAT-GRpep entered the brain after i.p. injection (Supplemental Figure 4E). For blood, we used flow cytometry instead, which also showed the presence of TAT-GRpep (Supplemental Figure $4 \mathrm{~F}$ ). These data support our conclusion that TAT-GRpep entered these tissues and was thus able to disrupt the GR-FKBP51 interaction, as shown by the coimmunoprecipitation experiments (Supplemental Figure 4, A-D).

We examined phosphorylation of $\mathrm{GR}$ at $\mathrm{S}_{211}$, which is within the region that interacts with FKBP51, and found that $S_{211}$ phosphorylation was decreased in both PTSD patients and fearconditioned mice. These data suggest that under pathological conditions, increased GR-FKBP51 complex formation could lead to decreased GR $\mathrm{S}_{211}$ phosphorylation as part of the pathophysiology of PTSD. Further experiments would be needed to determine the direction of causality between $\mathrm{GR} \mathrm{S}_{211}$ phosphorylation and GR binding to the FKBP5.

As mentioned earlier, we found decreased nuclear translocation of GR, which would be expected to affect gene transcription regulated by GR. Thus, we examined the expression of 14-3-3e, a known target of GR regulation. Both RNA and protein screening studies have previously identified $14-3-3 \varepsilon$ as a GR target protein (37). We did not perform further experiments to investigate the functional role of decreased $14-3-3 \varepsilon$ in PTSD. However, we speculate that $14-3-3 \varepsilon$ may be downregulated as part of the response to chronic physiological stress associated with PTSD symptoms. The 14-3-3 proteins function as a hub of protein signaling in the context of a different type of stress, cancer, at the cellular and biochemical level (61). More relevant to brain disorders, 14-3-3 proteins are involved in neurodevelopment and signaling, and have been implicated in the pathophysiology of schizophrenia and bipolar disorder, as well as number of neurological disorders (62). We are not aware of previous studies linking the 14-3-3 proteins with PTSD, so this observation requires further experiments to explain.

One limitation of our study is the use of peripheral blood as the human tissue for studying a brain disorder, and the cross-species translation to mouse tissue samples. We used peripheral blood to discover a protein biomarker for PTSD that we then targeted to successfully disrupt fear-conditioning memory formation and recall in the mouse. We validated the peripheral blood measurement of GR-FKBP51 levels as a proxy for brain by demonstrating similar findings in these 2 tissues in mice. TAT-GRpep peptide was also able to decrease GR-FKBP51 complex levels in both blood and brain tissue from mice (Supplemental Figure 4, A and B vs. C and D). These combined data provide strong evidence that the elevated protein complex we detected in blood from PTSD patients does indeed reflect a brain abnormality that when targeted in the mouse, can disrupt fear-memory paradigms relevant to PTSD in humans.

A final point for discussion is the heterogeneity of our human clinical subjects. This is a difficult issue for all human clinical studies. On one hand, having a more homogeneous sample will make biological conclusions simpler, but on the other hand, will be less representative of diverse clinical populations. The particular goals of each study influence the type of human population sample that is most informative. In the present study, we aimed to discover whether the GR-FKBP51 complex is involved in the biology of PTSD. Thus, a real-world sample would be most likely to be representative of the overall group of PTSD patients, and thus most likely to produce generally applicable results. As well, we have replicated our results in 2 completely separate cohorts of patients and controls, collected at 2 different times, in 2 different countries, by different clinical teams. Therefore, we argue that this heterogeneity of patients represents a strength, making our results more robust and generalizable to PTSD patients as a whole, not just a homogeneous subgroup.

Overall, our findings are significant for several reasons. First, we have evidence for a protein-protein interaction between the GR and FKBP51 proteins that represents an important molecular mechanism for modulating GR function under stress, in addition to the previously reported genetic interaction. Second, we have determined the binding sites for the GR-FKBP51 interaction and developed an interfering peptide as an experimental tool that also represents a potential treatment approach for PTSD. Third, because our interfering peptide can block the consolidation of fear memories, we propose that it or a therapeutic analog could be given to patients exposed to severe trauma, as a prophylaxis against the future emergence of PTSD. The protein complex could also be a treatment target for established PTSD symptoms and as a biochemical diagnostic marker for PTSD. Any of these advances would significantly improve on current clinical approaches to this important brain disorder. 


\section{Methods}

Human subject recruitment and clinical assessment. PTSD patients $(n=$ 22) were recruited from an inpatient ward and outpatient clinics at the Centre for Addiction and Mental Health (CAMH), and healthy controls $(n=22)$ were recruited with public advertisements. Traumatic memoryrelated symptoms were assessed with the civilian version of the PTSD checklist (PCL-C), which is a self-report questionnaire that permits the scoring of both severity of posttraumatic symptoms and the diagnosis of PTSD based on DSM-IV criteria (63). PTSD subjects underwent a structured evaluation of their diagnosis using the Mini International Neuropsychiatric Interview (MINI) (64). Exclusion criteria were acute or chronic physical illnesses or systemic treatment with corticosteroids. Control subjects were excluded if they had a psychiatric diagnosis (including substance abuse) or had any serious medical illness (requiring ongoing medical treatment). Two 10-mL tubes (BD Vacutainer, K2 EDTA) of peripheral blood were collected by venipuncture between 9 a.m. and 11 a.m., immediately after the interview. Lymphocytes were isolated using Ficoll-Paque PLUS (GE Healthcare Life Sciences) and stored at $-80^{\circ} \mathrm{C}$ until further processing. Kerry J. Ressler (McLean Hospital, Harvard Medical School, Belmont, Massachusetts) provided the human samples from the Grady Trauma Project in Atlanta.

Mice. Male C57BL/6 mice, aged 7 weeks, were purchased from Charles River Laboratories and given 1 week to acclimatize to the vivarium. Some animals were later subjected to implantation of cannula into either amygdala (AP - 1.5, LM - 3.0, DV - 4.8) or motor cortex (AP + 2.1, LM - 2.0, DV - 1.0). Following surgeries, animals were allowed 1 week to recover before additional experimental procedures. WeiDong Yong (Comparative Medical Center, Institute of Laboratory Animal Science, Chinese Academy of Medical Sciences \& Peking Union Medical College, Beijing, China) provided brain tissues from FKBP51 knockout mice.

Coimmunoprecipitation and Western blot. Coimmunoprecipitation and Western blot analyses were performed as previously described (2325). For coimmunoprecipitation, 500-1000 $\mu$ g solubilized protein was extracted from whole mouse brain or human lymphocytes, and incubated with primary antibody or control $\operatorname{IgG}(2-4 \mu \mathrm{g})$ for 4 hours at $4^{\circ} \mathrm{C}$, followed by the addition of $25 \mu \mathrm{L}$ protein $\mathrm{A} / \mathrm{G}$ plus agarose (Santa Cruz Biotechnology) for 12 hours. Pellets were washed, boiled for 5 minutes in SDS sample buffer, and subjected to SDS-PAGE. Total protein extract (50-100 $\mu \mathrm{g}$ ) was used as control in each experiment. After transfer of proteins into nitrocellulose, membranes were subject to Western blot with the primary antibodies. The protein level was quantified by densitometry (software from ImageLab, Bio-Rad).

The antibodies used were against GR (Santa Cruz Biotechnology, rabbit, catalog sc-1002; Proteintech Group, rabbit, catalog 24050-1AP), FKBP51 (Santa Cruz Biotechnology, rabbit, catalog sc-13983), FKBP52 (Santa Cruz Biotechnology, mouse, catalog sc-100758). The other antibodies were 14-3-3e (Santa Cruz Biotechnology, mouse, catalog sc-23957), $\beta$-Actin (Cell Signaling Technology, rabbit, catalog 4970), anti-Histone H3 (Abcam, rabbit, catalog ab1791), antiphospho-GR (Ser211) (Cell Signaling Technology, rabbit, catalog 4161) and anti- $\alpha$-tubulin (Sigma-Aldrich, mouse, catalog T8203).

GST fusion protein constructs and TAT-GRpep peptide. GST-fusion proteins encoding truncated GR fragments were amplified by PCR from full-length human cDNA clones. All constructs were sequenced to confirm the absence of PCR-generated errors. GST-fusion proteins were prepared from bacterial lysates with glutathione sepharose $4 \mathrm{~B}$ beads as instructed by the manufacturer (Amersham) (24). To construct GST-fusion proteins encoding truncated GR, cDNA fragments were amplified by using PCR with specific primers. To facilitate subcloning into the pGEX-4T3 vector, $5^{\prime}$ and $3^{\prime}$ oligonucleotides incorporating specific restriction enzyme sites were used. To confirm appropriate splice fusion and correct nucleotide sequences, all constructs were resequenced. TAT-GRpep peptide (AA sequence: GRKKRRQRRRPQSPWRSDLLIDENCLL) was synthesized by Biomatik.

Cued fear conditioning. Animals were first habituated to the apparatus for 5 minutes (Habitest, Colbourne Instruments). Animals were then exposed to 30 seconds of the cue (an in-house white light), which coterminated with 1 second of foot-shock $(0.5 \mathrm{~mA})$. The cue exposure and foot-shock were repeated 5 times, with 5 -minute inter-trial intervals. Following conditioning, animals were subjected to 2-5 days of extinction before assessment of cued fear memory, which consisted of 15 rounds of 30-second cue exposure, with 30-second inter-trial intervals (adapted from Andero et al.) (65). The expression of fear (in a different context from training) was assessed 24 hours after the last extinction session. The assessment consisted of a 3-minute period of habituation in the absence of CS, followed by a 3-minute trial of conditioned cues. The duration of freezing behaviors was recorded by an experimenter blind to the treatment group. Peptide was administered either 2 hours after conditioning or 2 hours before the behavioral assessment. The i.p. injection does was $3 \mathrm{nmol} / \mathrm{g}$ body weight. For amygdala and motor cortex administration, an injector was gently inserted into the guide cannula mounted onto the skulls of the animals. Control peptide or treatment peptide $(0.5 \mu \mathrm{L}$ at $10 \mathrm{mM}$ concentration) was slowly injected, and allowed to diffuse for 2 minutes before the injector was removed.

Nuclear protein isolation assay. Nuclear protein isolation assays were performed in mouse brain tissue using the Nuclear Protein Extraction Kit (Bio Basic Inc.). Nuclear protein isolation assays were performed in human lymphocytes using the NE-PER Nuclear and Cytoplasmic Extraction Reagents Kit (Thermo Fisher Scientific).

Immunohistochemistry. Mouse brains were dissected from designated groups 1 hour after i.p. injection of TAT-GRpep or saline, and then were fixed in $4 \%$ paraformaldehyde overnight, cryoprotected in $30 \%$ sucrose, and stored at $-80^{\circ} \mathrm{C}$ before further processing. Frozen coronal sections of $20-\mu \mathrm{m}$ thickness were cut using cryostat (Bright Instrument Co., model 5030). All sections were initially permeabilized with $0.1 \mathrm{M}$ PBS containing $0.1 \%$ Triton $\mathrm{X}-100$ for 10 minutes, and blocked for 1 hour with $1 \%$ bovine serum albumin in PBS at room temperature. Sections were incubated with anti-TAT antibody (1:500, Abcam, catalog ab43014) overnight at $4^{\circ} \mathrm{C}$ and secondary antibody for 1 hour at room temperature. The fluorescent secondary antibody conjugated to Alexa 488 (1:200, Life Technologies, catalog A21206) was used for detection of the primary antibody. DAPI was used to stain cell nuclei. Confocal images were acquired using a confocal microscope (Olympus FluoView FV1200) at $\times 10$ magnification.

Flow cytometry. Mouse whole blood was taken from the designated groups 1 hour after i.p. administration. Lymphocytes were isolated as indicated elsewhere in this paper. Briefly, cell pellets were fixed and washed twice in PBS, then resuspended in staining buffer (PBS with $4 \% \mathrm{FBS}$ and $0.05 \% \mathrm{Na}_{3} \mathrm{~N}$ ) at a concentration of 107 cells/mL. Lymphocytes were stained by anti-HIV1 TAT antibody (Abcam, catalog ab43016) using the Flow Cytometry Fixation/Permeabilization Kit (Biotium, catalog 23006). After incubation in the dark at room temperature for 30 minutes, cells were washed twice 
with the staining buffer. Cells were then strained $(35 \mu \mathrm{m})$ and read on the Cytoflex2 (Beckman Coulter). Results were analyzed using the FlowJo software.

Statistics. Densitometry of protein levels were normalized after being divided by the mean value of control levels from the same blot. Results for each sample were presented as the percentage of the control group. The independent samples $t$ test, 1-way ANOVA test followed by Dunnett's post hoc test, and 2-way ANOVA were used to compare different experimental groups. Data are shown as mean \pm SEM. The significance levels of $P$ less than $0.05, P$ less than 0.01 , or $P$ less than 0.001 were used for all analyses. Power and sample size analysis (PSS) as well as the 2-sample $t$ test (for human samples) were conducted using OriginPro 8 (OriginLab). All data were analyzed by SPSS (IBM Software Inc.), Prism 6 (GraphPad Software), and OriginPro 8 (OriginLab). Power and sample size analysis was conducted using $\mathrm{G}^{*}$ power (Heinrich-Heine-University Düsseldorf) (for mouse samples).

Study approval. All human subject procedures were conducted in accordance with the protocol (no. 002-2015) approved by the research ethics board at CAMH. All human subjects signed a written informed consent before participating and were free to withdraw from the study at any time. All animal experimental procedures were conducted in accordance with the protocols (no. 808) approved by the CAMH animal care committee.

Data availability. The data that support the findings of this study are available from the corresponding author upon reasonable request.

\section{Author contributions}

$\mathrm{HL}$ recruited human subjects at CAMH, conducted coimmunoprecipitation (co-ip), Western blot analysis using human samples, and affinity pull-down. PS conducted co-ip and Western blot analysis with the mouse samples, and co-ip using human depression samples. TKYL, AJ, JL, and SL conducted behavioral testing. CTGC, PS, and $\mathrm{DZ}$ conducted the flow cytometry analysis. FHFL performed the microscopy and image analysis. WDY provided brain tissues from FKBP51 knockout mice. SP supervised patient recruitment at CAMH. AHCW helped design the project, supervised patient recruitment at $\mathrm{CAMH}$, and wrote the paper. KJR provided the samples from the Grady Trauma Project in Atlanta, Georgia, and edited the paper. FL designed and supervised the project, and wrote the paper. HL is listed before PS because HL initiated this project.

\section{Acknowledgments}

We thank Longyu Dou in WeiDong Yong's lab for help with the FKBP51 knockout mice. The work is supported by an operating grant from the Canadian Institutes for Health Research (to FL; grant no. PJT 165978) and the Discovery Fund Seed Funding from the Centre for Addiction and Mental Health (to FL).

Address correspondence to: Fang Liu, Campbell Family Mental Health Research Institute, Centre for Addiction and Mental Health, 250 College Street, Toronto, Ontario M5T 1R8. Phone: 416.979.4659; Email:fang.liu@camh.ca.
1. American Psychiatric Association. Diagnostic and statistical manual of mental disorders. Arlington, VA: American Psychiatric Publishing; 2013.

2. Lawson NR. Posttraumatic stress disorder in combat veterans. JAAPA. 2014;27(5):18-22.

3. Johansen VA, Eilertsen DE, Nordanger D, Weisaeth L. Prevalence, comorbidity and stability of post-traumatic stress disorder and anxiety and depression symptoms after exposure to physical assault: an 8-year prospective longitudinal study. Nord J Psychiatry. 2013;67(1):69-80.

4. Smith TC, et al. PTSD prevalence, associated exposures, and functional health outcomes in a large, population-based military cohort. Public Health Rep. 2009;124(1):90-102.

5. Van Ameringen M, Mancini C, Patterson B, Boyle MH. Post-traumatic stress disorder in Canada. CNS Neurosci Ther. 2008;14(3):171-181.

6. Jonas DE, et al. Psychological pharmacological treatments for adults with posttraumatic stress disorder (PTSD). Comparative Effectiveness Reviews. 2013;(92): Report No. 13-EHC011-EF.

7. Bisson JI, Roberts NP, Andrew M, Cooper R, Lewis C. Psychological therapies for chronic post-traumatic stress disorder (PTSD) in adults. Cochrane Database Syst Rev. 2013;(12):CD003388.

8. Hetrick SE, Purcell R, Garner B, Parslow R. Combined pharmacotherapy and psychological therapies for post traumatic stress disorder (PTSD). Cochrane Database Syst Rev. 2010;(7):CD007316.

9. Murdoch M, et al. Long-term outcomes of disability benefits in US veterans with posttraumatic stress disorder. Arch Gen Psychiatry. 2011;68(10):1072-1080.
10. Zatzick D, et al. A national US study of posttraumatic stress disorder, depression, and work and functional outcomes after hospitalization for traumatic injury. Ann Surg. 2008;248(3):429-437.

11. Shalev AY. Posttraumatic stress disorder and stress-related disorders. Psychiatr Clin North Am. 2009;32(3):687-704.

12. Klengel T, et al. Allele-specific FKBP5 DNA demethylation mediates gene-childhood trauma interactions. Nat Neurosci. 2013;16(1):33-41.

13. Scammell JG, Denny WB, Valentine DL, Smith DF. Overexpression of the FK506-binding immunophilin FKBP51 is the common cause of glucocorticoid resistance in three New World primates. Gen Comp Endocrinol. 2001;124(2):152-165.

14. Binder EB, et al. Association of FKBP5 polymorphisms and childhood abuse with risk of posttraumatic stress disorder symptoms in adults. JAMA. 2008;299(11):1291-1305.

15. Mehta D, et al. Using polymorphisms in FKBP5 to define biologically distinct subtypes of posttraumatic stress disorder: evidence from endocrine and gene expression studies. Arch Gen Psychiatry. 2011;68(9):901-910.

16. Szyf M. How do environments talk to genes? Nat Neurosci. 2013;16(1):2-4.

17. Gallo LI, Lagadari M, Piwien-Pilipuk G, Galigniana MD. The 90-kDa heat-shock protein (Hsp90)-binding immunophilin FKBP51 is a mitochondrial protein that translocates to the nucleus to protect cells against oxidative stress. J Biol Chem. 2011;286(34):30152-30160.

18. Zhang X, Clark AF, Yorio T. FK506-binding protein 51 regulates nuclear transport of the glucocorticoid receptor beta and glucocorti- coid responsiveness. Invest Ophthalmol Vis Sci. 2008;49(3):1037-1047.

19. Wochnik GM, Rüegg J, Abel GA, Schmidt U, Holsboer F, Rein T. FK506-binding proteins 51 and 52 differentially regulate dynein interaction and nuclear translocation of the glucocorticoid receptor in mammalian cells. J Biol Chem. 2005;280(6):4609-4616.

20. Zhang L, et al. Loss of FKBP5 impedes adipocyte differentiation under both normoxia and hypoxic stress. Biochem Biophys Res Commun. 2017;485(4):761-767.

21. Stechschulte LA, et al. FKBP51 null mice are resistant to diet-induced obesity and the PPAR $\gamma$ agonist rosiglitazone. Endocrinology. 2016;157(10):3888-3900.

22. Pratt WB. Transformation of glucocorticoid and progesterone receptors to the DNA-binding state. J Cell Biochem. 1987;35(1):51-68.

23. Su P, et al. A dopamine D2 receptor-DISC1 protein complex may contribute to antipsychoticlike effects. Neuron. 2014;84(6):1302-1316.

24. Lee FJ, Pei L, Moszczynska A, Vukusic B, Fletcher PJ, Liu F. Dopamine transporter cell surface localization facilitated by a direct interaction with the dopamine D2 receptor. EMBO J. 2007;26(8):2127-2136.

25. Pei L, et al. Uncoupling the dopamine D1-D2 receptor complex exerts antidepressant-like effects. Nat Med. 2010;16(12):1393-1395.

26. Golub Y, Mauch CP, Dahlhoff M, Wotjak CT. Consequences of extinction training on associative and non-associative fear in a mouse model of posttraumatic stress disorder (PTSD). Behav Brain Res. 2009;205(2):544-549. 
27. Brinks V, de Kloet ER, Oitzl MS. Strain specific fear behaviour and glucocorticoid response to aversive events: modelling PTSD in mice. Prog Brain Res. 2008;167:257-261.

28. Siegmund A, Wotjak CT. A mouse model of posttraumatic stress disorder that distinguishes between conditioned and sensitised fear. J Psychiatr Res. 2007;41(10):848-860.

29. Mahan AL, Mou L, Shah N, Hu JH, Worley PF, Ressler KJ. Epigenetic modulation of Homerla transcription regulation in amygdala and hippocampus with pavlovian fear conditioning. J Neurosci. 2012;32(13):4651-4659.

30. Anbalagan M, Huderson B, Murphy L, Rowan BG. Post-translational modifications of nuclear receptors and human disease. Nucl Recept Signal. 2012;10:e001.

31. Oakley RH, Cidlowski JA. The biology of the glucocorticoid receptor: new signaling mechanisms in health and disease. JAllergy Clin Immunol. 2013;132(5):1033-1044.

32. Wallace AD, Cidlowski JA. Proteasome-mediated glucocorticoid receptor degradation restricts transcriptional signaling by glucocorticoids. J Biol Chem. 2001;276(46):42714-42721.

33. Webster JC, Jewell CM, Bodwell JE, Munck A, Sar M, Cidlowski JA. Mouse glucocorticoid receptor phosphorylation status influences multiple functions of the receptor protein. J Biol Chem. 1997;272(14):9287-9293.

34. Miller AL, et al. p38 Mitogen-activated protein kinase (MAPK) is a key mediator in glucocorticoid-induced apoptosis of lymphoid cells: correlation between p38 MAPK activation and site-specific phosphorylation of the human glucocorticoid receptor at serine 211. Mol Endocrinol. 2005;19(6):1569-1583.

35. Wang Z, Frederick J, Garabedian MJ. Deciphering the phosphorylation "code" of the glucocorticoid receptor in vivo. JBiol Chem. 2002;277(29):26573-26580.

36. Jonas DE, et al. Psychological and Pharmacological Treatments for Adults With Posttraumatic Stress Disorder (PTSD). Rockville, MD; 2013.

37. Ayyar VS, Almon RR, DuBois DC, Sukumaran S, $\mathrm{Qu}$ J, Jusko WJ. Functional proteomic analysis of corticosteroid pharmacodynamics in rat liver: Relationship to hepatic stress, signaling, energy regulation, and drug metabolism. J Proteomics. 2017;160:84-105.

38. Phuc Le P, et al. Glucocorticoid receptordependent gene regulatory networks. PLoS Genet. 2005;1(2):e16.

39. Ressler KJ, et al. Post-traumatic stress disorder is associated with PACAP and the PAC1 receptor. Nature. 2011;470(7335):492-497.

40. Gillespie CF, et al. Trauma exposure and stress-related disorders in inner city primary care patients. Gen Hosp Psychiatry. 2009;31(6):505-514

41. Lukic I, et al. Accumulation of cytoplasmic glucocorticoid receptor is related to elevation of FKBP5 in lymphocytes of depressed patients. JMol Neurosci. 2015;55(4):951-958.

42. Tatro ET, Everall IP, Kaul M, Achim CL. Modulation of glucocorticoid receptor nuclear translocation in neurons by immunophilins FKBP51 and FKBP52: implications for major depressive disorder. Brain Res. 2009;1286:1-12.

43. Yehuda R, LeDoux J. Response variation following trauma: a translational neuroscience approach to understanding PTSD. Neuron. 2007;56(1):19-32.

44. Criado-Marrero M, Rein T, Binder EB, Porter JT, Koren J, Blair LJ. Hsp90 and FKBP51: complex regulators of psychiatric diseases. Philos Trans $R$ Soc Lond, B, Biol Sci. 2018;373(1738):20160532.

45. Brown V, Liu F. Intranasal delivery of a peptide with antidepressant-like effect. Neuropsychophar macology. 2014;39(9):2131-2141.

46. Zhai D, et al. Blocking GluR2-GAPDH ameliorates experimental autoimmune encephalomyelitis. Ann Clin Transl Neurol. 2015;2(4):388-400.

47. Yehuda R, Southwick SM, Krystal JH, Bremner D, Charney DS, Mason JW. Enhanced suppression of cortisol following dexamethasone administration in posttraumatic stress disorder. Am J Psychiatry. 1993;150(1):83-86.

48. Yehuda R, Golier JA, Yang RK, Tischler L. Enhanced sensitivity to glucocorticoids in peripheral mononuclear leukocytes in posttraumatic stress disorder. Biol Psychiatry. 2004;55(11):1110-1116.

49. Raison CL, Miller AH. When not enough is too much: the role of insufficient glucocorticoid signaling in the pathophysiology of stress-related disorders. Am J Psychiatry. 2003;160(9):1554-1565.

50. van Zuiden M, et al. Glucocorticoid sensitivity of leukocytes predicts PTSD, depressive and fatigue symptoms after military deployment: A prospective study. Psychoneuroendocrinology. 2012;37(11):1822-1836.

51. Nierenberg AA, Feinstein AR. How to evaluate a diagnostic marker test. Lessons from the rise and fall of dexamethasone suppression test. JAMA. 1988;259(11):1699-1702.

52. Zannas AS, et al. Epigenetic upregulation of FKBP5 by aging and stress contributes to NF- $\mathrm{kB}$-driven inflammation and cardiovascular risk. Proc Natl
Acad Sci USA. 2019;116(23):11370-11379.

53. Menke A, et al. Childhood trauma dependent anxious depression sensitizes HPA axis function. Psychoneuroendocrinology. 2018;98:22-29.

54. Binder EB, et al. Polymorphisms in FKBP5 are associated with increased recurrence of depressive episodes and rapid response to antidepressant treatment. Nat Genet. 2004;36(12):1319-1325.

55. Cheung-Flynn J, Roberts PJ, Riggs DL, Smith DF. C-terminal sequences outside the tetratricopeptide repeat domain of FKBP51 and FKBP52 cause differential binding to Hsp90. J Biol Chem. 2003;278(19):17388-17394

56. Howard KJ, Holley SJ, Yamamoto KR, Distelhorst CW. Mapping the HSP90 binding region of the glucocorticoid receptor. J Biol Chem 1990;265(20):11928-11935.

57. Xu M, Dittmar KD, Giannoukos G, Pratt WB, Simons SS. Binding of hsp90 to the glucocorticoid receptor requires a specific 7-amino acid sequence at the amino terminus of the hormone-binding domain. JBiol Chem. 1998;273(22):13918-13924.

58. Lee FJ, et al. Dual regulation of NMDA receptor functions by direct protein-protein interactions with the dopamine D1 receptor. Cell. 2002;111(2):219-230.

59. Arriza JL, et al. Cloning of human mineralocorticoid receptor complementary DNA: structural and functional kinship with the glucocorticoid receptor. Science. 1987;237(4812):268-275

60. Pippal JB, Fuller PJ. Structure-function relationships in the mineralocorticoid receptor. $J \mathrm{Mol}$ Endocrinol. 2008;41(6):405-413.

61. Pennington KL, Chan TY, Torres MP, Andersen JL. The dynamic and stress-adaptive signaling hub of 14-3-3: emerging mechanisms of regulation and context-dependent protein-protein interactions. Oncogene. 2018;37(42):5587-5604.

62. Foote M, Zhou Y. 14-3-3 proteins in neurological disorders. Int J Biochem Mol Biol. 2012;3(2):152-164.

63. Ruggiero KJ, Del Ben K, Scotti JR, Rabalais AE. Psychometric properties of the PTSD Checklist-Civilian Version. J Trauma Stress. 2003;16(5):495-502.

64. Sheehan DV, et al. The Mini-International Neuropsychiatric Interview (M.I.N.I.): the development and validation of a structured diagnostic psychiatric interview for DSM-IV and ICD-10. J Clin Psychiatry. 1998;59(Suppl 20):22-33.

65. Andero R, Heldt SA, Ye K, Liu X, Armario A Ressler KJ. Effect of 7,8-dihydroxyflavone, a small-molecule TrkB agonist, on emotional learning. Am J Psychiatry. 2011;168(2):163-172. 